\title{
TelefonSeelsorge: ihr Angebot, ihre Nutzer und die Anforderungen an die Mitarbeitenden
}

Ruth Belzner

\author{
Jeder Kontakt in der TelefonSeelsorge erhält seine Gestalt durch 3 komplexe \\ Determinanten: die TelefonSeelsorge als Organisation mit ihrem Selbstverständ- \\ nis, ihrer Struktur und mit den Möglichkeiten und Grenzen des genutzten Medi- \\ ums (Telefon, Mail, Chat), die Ratsuchende bzw. den Ratsuchenden mit dem \\ aktuellen Thema und Anliegen, mit ihrem bzw.seinem Kommunikationsstil und \\ Erwartungen an die TelefonSeelsorge, und den Seelsorger bzw. die Seelsorgerin \\ mit der ihm bzw. ihr eigenen Persönlichkeit und Identität.
}

\section{Die Organisation TelefonSeelsorge}

Angesichts der hohen Suizidzahlen in Berlin wurde dort 1956 vom Theologen und Psychotherapeuten Klaus Thomas eine ehrenamtlich arbeitende, telefonisch erreichbare „Ärztliche Lebensmüdenbetreuung“ gegründet. Im Jahr drauf entstand in Kassel eine weitere ehrenamtlich arbeitende telefonische Einrichtung der Suizidprävention. Dort wurde der Name „Telefonseelsorge“ geprägt, den alle in Folge gegründeten Stellen übernahmen. Bis 1997 war die Telefonseelsorge ein Verbund voneinander unabhängiger Stellen.

Seit Juli 1997 ist die TelefonSeelsorge über 2 bundesweit einheitliche Freecall-Nummern erreichbar (08001110111 und 08001110222 - der TelefonSeelsorge von der Deutschen Telekom unentgeltlich zur Verfügung gestellt), seit 1999 ist die „TelefonSeelsorge“ eine gesetzlich geschützte Wortmarke. Aus einem Verbund von TelefonSeelsorgeStellen wurde eine hochvernetzte Organisation. Anrufende werden aufgrund ihres Standortes „ihrer“ Stelle bzw. Organisationseinheit (Verbund mehrerer Stellen, der gemeinsam deren Einzugsbereiche versorgt) zugeordnet. Die technische Interdependenz und die Tatsache, dass nun von Nutzern nicht mehr gezielt eine präferierte Stelle, sondern „die TelefonSeelsorge“ in Anspruch genommen wird, führte zu einem hohen Abstimmungs-, Regelungs- und Steuerungsbedarf in der Gesamtorganisation.

Das Selbstverständnis der TelefonSeelsorge ist in ihrem für alle Stellen verbindlichen Organisationshandbuch [1] so formuliert: „Die TelefonSeelsorge bietet Hilfe suchenden Menschen qualifizierte und vertrauliche Seelsorge und Beratung an, vorurteilsfrei und offen. Das Angebot der TelefonseelsorgerInnen besteht im Zuhören und Klären, im Ermutigen und Mittragen, in der Unterstützung bei Entscheidungsfindung und im Hinweis auf spezifische Hilfsangebote. Sie macht Mut zum Leben und stärkt Menschen in ihrem Glauben und Hoffen. Wenn Begegnen, Klären, Halt geben oder Begleiten nicht möglich ist oder wenn Grenzen und Würde der Mitarbeitenden verletzt werden, beendet TelefonSeelsorge den Kontakt.“

Die Offenheit des Angebotes braucht die Begrenzung. Das war in der Realität wohl schon immer so, wurde allerdings erst 2006 zum ersten Mal so in den „Merkmalen qualitätsorientierter TelefonSeelsorge " formuliert und von dort in das Handbuch übernommen. Neben den persönlichen Grenzen der Mitarbeitenden schützt diese Begrenzung auch das Angebot als solches.

Für die Ratsuchenden bedeutet TelefonSeelsorge: Man kann sie jederzeit, von überall und spontan nutzen, sofern man ein Telefon oder Handy oder Zugang zum Internet hat. Einschränkend ist allerdings zu sagen, dass nicht jeder Anrufversuch auf eine freie Leitung trifft, dass man Glück und Ausdauer braucht, um einen Chattermin zu ergattern, und dass Mails nicht immer innerhalb der eigentlich zugesagten 48-Stunden-Frist beantwortet werden können. Für alle drei Medien gilt: Die Nachfrage ist vielfach größer, als es die Ressourcen sind, die die TelefonSeelsorge mit ca. 8000 ehrenamtlich Mitarbeitenden in 105 Stellen zur Verfügung hat.

Man muss keine Information preisgeben, die einen als Person identifizierbar machen könnte, und man wird nichts gefragt, das für das eigentliche Anliegen unerheblich ist. Der Kontakt ist in allen Medien (Telefon, Mail und Chat) bestmöglich datengeschützt. Das garantiert, dass man hier offen über seine Situation und Befindlichkeit sprechen kann, auch über akute Suizidalität, ohne fürchten zu müssen, dass gegen den eigenen Willen interveniert wird. Und grundsätzlich kann man jeden Kontakt jederzeit abbrechen, ohne Konsequenzen oder das Recht auf einen nächsten Kontakt zu verlieren. 
Man braucht keine Indikation, man muss kein Problem oder Ziel definieren, es reicht, „einfach mal reden“ (eine häufig gehörte Formulierung) zu wollen. Häufig geht es „nur“ um die tägliche Dosis Kontakt für einen sehr einsamen Menschen - ein Anliegen, das ein Mitarbeiter kürzlich in einer Gesprächsnotiz so beschrieb: „In den 29:00 Minuten unseres Gespräches war er nicht allein und ein bisschen ,wir' hat existiert." Allerdings: Immer wieder zeigen sich auch nach diesem scheinbar harmlosen Einstieg dann im Gespräch Menschen in extrem belastenden Lebenssituationen oder akuten schweren Krisen. Mit dieser Bandbreite wissen die gut ausgebildeten und durch Supervision begleiteten Mitarbeitenden umzugehen.

\section{Die Ratsuchenden}

Die TelefonSeelsorge erfasst für alle Seelsorge-Kontakte statistische Daten: Zeitpunkt und Dauer des Kontakts, Geschlecht, (geschätztes) Alter, (vermutete) Lebensform und Erwerbsstatus, ob psychisch erkrankt, ob Suizidalität eine Rolle spielt, ob man es für einen Erst- oder wiederholten Kontakt hält, und schließlich sind aus einer vorgegebenen Themenliste bis zu drei Items auszuwählen. Diese Daten erlauben keine Rückschlüsse auf die Zahl der dahinterstehenden Personen, denn mindestens $70 \%$ aller Gespräche und Chats finden mit Menschen statt, die wiederholt den Kontakt suchen. Die Daten zeigen aber, in welchen Lebenssituationen und mit welchen Themen Ratsuchende sich bevorzugt an die TelefonSeelsorge wenden.

Mehr als $30 \%$ aller Gespräche führen wir mit Menschen, von denen wir wissen, dass sie psychisch erkrankt sind - Tendenz steigend. Über $60 \%$ der Anrufe kommen von Alleinlebenden. Die größte Alterskohorte ist mit 30\% die der 50-bis-59-Jährigen. Mit $27 \%$ ist „erwerbsunfähig“ der am häufigsten genannte Erwerbsstatus. In $8 \%$ aller telefonischen Kontakte kommt Suizidalität zu Sprache. Auffallend ist, dass in $80 \%$ der Gespräche mit suizidalen Inhalten eine psychische Erkrankung mit im Spiel ist und in der Hälfte dieser Fälle explizit eine depressive Stimmung genannt wird.

Den engen Zusammenhang zwischen psychischer Erkrankung, Hoffnungslosigkeit und Suizidalität haben Klein, Strietholt und Stuckstätte in einer Auswertung aller Gesprächsdaten von Juli 2014 bis Juni 2015 festgestellt: „Suizidale Absichten stehen häufig - nicht immer - in Zusammenhang mit psychischen oder physischen (chronischen) Erkrankungen mit fehlender Heilungsaussicht. Sowohl in Anrufen von Personen mit Suizidgedanken als auch bei einem früheren Suizidversuch ist das seelische Befinden mit 59,2\% bzw. 57,1 \% ein zentrales Gesprächsthema.“ [2, S. 130] Und: „Das körperliche Befinden von Personen mit Suizidabsichten bzw. Suizidgedanken wurde mit steigendem Alter immer häufiger thematisiert. Ebenfalls stieg mit höherem Alter die Relevanz des Themas Einsamkeit und Isolation kontinuierlich an.“ [2, S. 131]. Sie schluss- folgern: „Es ist davon auszugehen, dass die häufig mangelhafte Versorgungslage psychisch kranker Menschen in Deutschland dazu führt, dass Institutionen wie die TS phasenweise oder über einen langen Zeitraum eine zentrale Instanz in der Begleitung dieser Zielgruppen sind. " [2, S. 132] Das deckt sich mit den Erfahrungen meiner Mitarbeitenden am Telefon und auch im Chat.

Zu Mail und Chat gibt es bisher noch keine vergleichbare Studie. Die Daten, die wir erheben, machen allerdings deutlich: Insbesondere der Chat ist das Medium der Jüngeren in schweren Krisen: 57,7\% der Kontakte kommt von Menschen zwischen 15 und 29 Jahren und in $42 \%$ der Chats spielt Suizidalität eine Rolle, auch wenn sie in „nur“ $10 \%$ zentral thematisiert wird.

Viele einsame Menschen suchen in ihren Anrufen in erster Linie ihre „tägliche Dosis Kontakt“, die Themen der Gespräche sind oft unspektakulär. Was hier von den Mitarbeitenden gefordert ist, ist Zuwendung und Interesse - und die Fähigkeit, das Gespräch auch wieder zu beenden.

Andere Anforderungen stellte dieses über eine Stunde dauerndes Gespräch um 01:00 Uhr nachts mit einem Erstanrufer, dargestellt anhand der Gesprächsnotiz der Mitarbeiterin: „Der Anrufer, 32, lebt in einer Beziehung, hat eine Stelle im Rheinland (Wochenpendler) und steht nach der Probezeit vor der Entscheidung, umzuziehen. Die Partnerin würde mitgehen, fände sicher auch dort eine Stelle, eine Wohnung bekämen sie. Eigentlich klar, wie er sich vernünftigerweise entscheiden sollte. Unter seinem Zögern und Zweifeln (von dem sein Umfeld, insbesondere die Freundin, schon genervt ist) werden nach und nach seine tiefsitzenden Ängste und Selbstzweifel deutlich. Er hat privat (nicht beruflich) fürchterliche Angst vor Entscheidungen, die auch andere Menschen betreffen, will alles bis ins Kleinste durchdenken, vorhersehen, planen... Ihm wird deutlich, wie sehr ihn seine Ängste belasten und behindern und er will sich therapeutische Hilfe suchen. Er ist dankbar für die Zeit, die wir uns genommen haben, um wirklich zu verstehen, um was es geht. Ob er nun den Mietvertrag für die Wohnung im Rheinland unterschreibt, das blieb offen - ich habe nicht mehr nachgefragt."

Hier ist zu dem Interesse auch klärendes Nachfragen, Hypothesen-Anbieten und Verstandenes-Zusammenfassen gefordert - und nicht Auf-ein-Ergebnis-Drängen.

Die folgende Notiz zu einem 30-minütigen nächtlichen Gespräch mit einem akut suizidalen Menschen zeigt, dass auch Mut wichtig sein kann: „Der erste Satz des Anrufers: ,Mir geht es ganz schlecht' (das höre ich auch an seiner Stimme). Er wollte sich heute das Leben nehmen und hat es nicht geschafft. Dabei war die Idee ,wenn's gar nicht mehr geht, dann bringe ich mich um' immer sein Trost. Und nicht mal den hat er jetzt noch, meint er. Er kann mit niemandem in seinem Umfeld über seinen Todeswunsch 
sprechen. Er weiß, dass er Angehörigen und Freunden Schmerz zufügen würde, aber sein eigener Schmerz (er kann sich nicht an gute Zeiten erinnern) ist für ihn unerträglich. Ich erfrage dann doch ein paar Fakten: 29 Jahre, Ingenieur, seit einem Jahr wegen schwerster Depressionen krankgeschrieben, mehrmals in der Klinik, mit jeweils kurzer Besserung. Ich brauche Mut dazu, biete ihm dann doch folgendes Bild an: Sein Schmerz ist ein Gefängnis, in dem die Tür ,Suizid' ihm das Aushalten erleichtert. Und jetzt merkt er, er kriegt die Tür nicht auf. Doch die Tür bleibt ja (trotz seiner heutigen Erfahrung) und dass sie ihm derzeit verschlossen ist, gibt ihm die Chance, doch noch nach anderen Türen zu suchen. Ein erster Schritt wäre, sich an den Krisendienst zu wenden, der am nächsten Morgen wieder erreichbar ist. Das Bild mit den Türen findet er hilfreich. Und er verabschiedet sich dann, wie er sagt und wie auch zu hören ist, ruhiger und weniger verzweifelt. Angerufen hat er ganz spontan, ohne sagen zu können, was er sich erhofft. Als er im Internet nach Suizidmethoden gesucht hat, kam immer wieder der Hinweis, sich an die TelefonSeelsorge zu wenden und dann hat er es halt mal probiert.“

Eine weitere Anforderung zeigt ein 35-minütiges Gespräch, das ein Mitarbeiter mit einer ca. 50-jährigen wiederholt Anrufenden führte: „Anruferin ist völlig verzweifelt. Sie leidet unter einer posttraumatischen Belastungsstörung, verursacht durch Vergewaltigungen durch ihren Vater. Sie habe alles getan, um dieses Trauma zu überwinden und sei seit über 30 Jahren in Behandlung, hat 3 Suizidversuche hinter sich. (...) Sie komme sich vor wie ein Zombie und verstehe nicht, dass Gott kein Einsehen mit ihr habe. Ich finde es schwer, ihr Leiden auszuhalten. Aber sie bedankt sich sehr fürs Zuhören.“

„Aushalten“ ist ein wichtiges Stichwort für viele Gespräche und Online-Kontakte. Das gilt auch da, wo man als Seelsorgerln eine Veränderungsmöglichkeit sieht und Ratsuchende diese Sichtweise nicht übernehmen. Nichtveränderung disqualifiziert diese Menschen nicht für die TelefonSeelsorge; im Gegenteil, hier ist ein Ort, wo jemand anderer es immer wieder mit ihnen aushält.

\section{Die Mitarbeitenden}

Wer sind diese „anderen“, die Menschen, die am Telefon sitzen, chatten und Mails beantworten?

Zu den ehrenamtlich Mitarbeitenden gibt es aktuell keine bundesweiten soziografischen Daten. Eine Umfrage 2011 zu „Engagement und Zufriedenheit“, an der 2183 Ehrenamtliche teilnahmen, und in der solche Daten erhoben worden, legt allerdings nahe, dass das, was ich über die Mitarbeitenden der Würzburger Stelle sagen kann, repräsentativ ist: $25 \%$ Männer und 75 \% Frauen zwischen 25 und 80 Jahren. Der Altersmedian liegt bei 62 Jahren. $80 \%$ der Mitarbeitenden leben in festen Beziehungen, die Hälfte hat einen Hochschulabschluss, weitere $15 \%$ Abitur. Sie kommen aus einem anderen sozialen Milieu als viele der Anrufenden, und das im Blick zu haben ist wichtig für die Ausbildung.

„Sicherlich ist eine explizite Sensibilisierung der TS-Mitarbeitenden für die Existenz der Barrieren und Abstoßungseffekte zwischen den Milieus sinnvoll und notwendig." [3, S. 157] Das bezieht sich nicht nur auf den Umgang mit Sprache, die seitens der Mitarbeitenden möglichst wenig milieuspezifisch und „leicht“ sein sollte, sondern auch auf ein Bewusstsein für die möglicherweise ganz andere Sicht der Ratsuchenden auf sich und ihre Lage, als sie der/die Mitarbeitende hätte: „Für gelingende Gespräche in der TS ist es daher wichtig, sich nicht nur die eigene Sichtweise auf eine soziale Notlage bewusst zu machen, sondern auch die Sichtweisen der Anrufenden zu verstehen, von denen maßgeblich abhängt, welche Art von Hilfe möglich und nötig ist. " [3, S. 159]

Von den soziografischen Daten und ihrer Bedeutung für die Schulung der Mitarbeitenden hin zur Frage der Persönlichkeiten: Anzunehmen ist, dass auch am Telefon die Persönlichkeit des/der Mitarbeitenden ein wesentlicher Wirkfaktor ist (kein therapeutischer, wohl aber ein Halt gebender, klärender, ermutigender ...). Insofern ist es wichtig, was sich über die Persönlichkeiten der Mitarbeitenden und über die Wirkung der Ausbildung in dieser Hinsicht sagen lässt. Eine empirische Studie der Universität Heidelberg 2014/15 ermöglicht uns nun entsprechende Aussagen. [4] Auszubildende an 27 Stellen füllten jeweils zu Beginn, Mitte und Abschluss der Ausbildung umfangreiche Fragebögen, zu Persönlichkeitsstil, Lebenszufriedenheit, Bindungsverhalten und Mentalisierung aus. 180 von 256 nahmen an allen 3 Messzeitpunkten teil.

In Bezug auf Persönlichkeitsstile zeigten die Teilnehmenden deutlich höhere Werte in Extraversion und Offenheit für Erfahrungen und leicht niedrigere Werte in Neurotizismus als eine Vergleichsgruppe mit gleicher Alters- und Geschlechtsverteilung. Zudem sind sie signifikant weniger bindungsvermeidend und sie zeigen eine hohe Lebenszufriedenheit. Zur Mentalisierung gibt es Vergleichsmöglichkeiten bisher nur mit Patienten in stationärer Psychotherapie, hier haben die Auszubildenden von Anfang an insbesondere eine ausgeprägte Bereitschaft zur Selbstreflexion. Signifikante Effekte hatte die Ausbildung bei der Bindungsangst; der schon geringe Anfangswert nahm weiter $a b$.

Zu den interpersonellen Motiven fanden Dinger \& Reek [4] heraus: „Im Vergleich mit der deutschen Allgemeinbevölkerung zeigte sich, dass die TS-Auszubildenden mehr nach Harmonie und Akzeptanz im Kontakt mit anderen streben, aber deutlich weniger durch das Streben nach Selbstsicherheit, Durchsetzung der eigenen Interessen, Selbstbezogenheit, Verschlossenheit und Unterordnung 
motiviert sind. Eine nachfolgende Clusterzentrenanalyse zeigte, dass die zukünftigen TS-BeraterInnen alle vorwiegend freundlich-zugewandte interpersonelle Motive haben, sich aber in der Ausprägung des Dominanzmotives unterschieden. Hier finden sich drei Untergruppen. Diese können hinsichtlich der Ausprägung ihrer interpersonellen Motive als ,altruistisch-submissiv“, ,helfend-beeinflussend ' und als, freundlich zugewandt' bezeichnet werden.“ [4, S. 385]

Die deutlichste Wirkung hatte die Ausbildung auf die Motive „Unterwürfigkeit“ und „Altruismus“: „Die TeilnehmerInnen berichteten nach Ende der Ausbildung, dass sie in ihren sozialen Beziehungen weniger stark durch ein Streben nach Unterordnung (z. B. das machen, was andere sagen) und Altruismus (z. B. die Bedürfnisse von anderen vor die eigenen stellen) motiviert waren.“ [4, S.492]

Das mag überraschend wirken, wenn man annimmt, dass sie lernen wollen und sollen, hilfreich zu sein. Es erklärt sich als Ausbildungserfolg, wenn man zugrunde legt, dass Mitarbeitende vor allem dann am Telefon und im Chat ein hilfreiches Gegenüber sind, wenn sie ein Gespräch auch steuern und fokussieren können (und nicht einfach den Gedankenschleifen der Ratsuchenden folgen) und dabei auch auf sich und ihre Grenzen achten.

Was für die TelefonSeelsorge bei der Auswahl, Ausbildung und Supervision der Mitarbeitenden zentrale Bedeutung hat, lässt sich so zusammenfassen: Es geht um persönliche Qualitäten und Potenziale. Mitarbeitende brauchen sowohl Einfühlungsvermögen als auch inneren Abstand. Sie benötigen Geduld, gleichzeitig Struktur und Klarheit, sowie einiges an psychosozialem Grundwissen, und dennoch einen offenen, nicht diagnostizierenden Blick auf den anderen Menschen. Wichtig ist zudem eine zuversichtliche Haltung zum Leben, Verständnis für die Hilf- und Hoffnungslosigkeit von Anrufenden, Interesse an anderen Menschen, ein feines Gespür für die Grenze zur indiskreten Neugier, eine sehr reflektierte Gesprächsführung und auch die Unbekümmertheit, einfach den eigenen Impulsen zu folgen.

Bei der Arbeit in der TelefonSeelsorge bewegt man sich in einem sehr spannenden und spannungsreichen Umfeld. Vieles lässt sich in der Ausbildung, in Fortbildungen und Supervisionen schulen und festigen, aber Voraussetzung ist, dass Menschen die Fähigkeit, sich in einem solchen spannungsreichen Feld zu bewegen, grundsätzlich mitbringen.
FAZIT

Es bleibt zu hoffen, um der Ratsuchenden willen, aber auch für die Gesellschaft als Ganze (denn ausgebildete TelefonseelsorgerInnen gehen auch außerhalb der Institution bewusster und klarer in Gesprächssituationen als vorher), dass sich immer ausreichend Menschen finden, die bereit und befähigt sind, hier mitzuarbeiten; und dass die Träger vor Ort und die Kirchen die TelefonSeelsorge als ein unverzichtbares, für viele Menschen lebenserhaltendes Angebot sehen und absichern.

Interessenkonflikt

Die Autorin gibt an, dass kein Interessenkonflikt besteht.

\section{Autorinnen/Autoren}

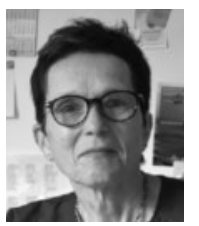

\section{Ruth Belzner}

Dipl.-Psych. Psychologiestudium in Düsseldorf und München. Ausbildung in der Evangelischen TelefonSeelsorge München und ehrenamtliche Mitarbeit. Mitarbeiterin des Centrum Mission/Eine Welt (damals „Missionswerk“) der Evang.-Luth. Kirche Bayern als Beauftragte für Kirchliche Frauenarbeit im Kirchenkreis Madang (Papua-Neuguinea). Seit 1996 Leiterin der TelefonSeelsorge Würzburg/Main-Rhön. Masterstudium Sozialmanagement an der EFH Darmstadt. 2004-2019 im Vorstand der Evangelischen Konferenz für TelefonSeelsorge und Offene Tür e. V.

\section{Korrespondenzadresse}

\author{
Dipl.-Psych. Ruth Belzner \\ MA Sozialmanagement \\ Telefonseelsorge Würzburg \\ PF 110812 \\ 97034 Würzburg \\ belzner@telefonseelsorge-wuerzburg.de
}

\section{Literatur}

[1] Handbuch der TelefonSeelsorge. Im Internet: https://www. telefonseelsorge.de/sites/default/files/TS_Organisationshandbuch_2014_web.pdf; Stand: März 2019

[2] Klein M, Strietholt R, Stuckstätte EC. Der Seelsorgeauftrag der TelefonSeelsorge. In: Hauschildt E, Blömeke BD, Hrsg. TelefonSeelsorge interdisziplinär. Göttingen: Vandenhoeck \& Ruprecht; 2015 
[3] Kläden T. TelefonSeelsorge und soziale Milieus. In: Hauschildt E, Blömeke BD, Hrsg. TelefonSeelsorge interdisziplinär. Göttingen: Vandenhoeck \& Ruprecht; 2015

[4] Dinger U, Reek I. Effekte der Seelsorgeausbildung Ehrenamtlicher. Pastoraltheologie mit GPM 2017; 106: 385-492

Bibliografie

DOI https://doi.org/10.1055/a-0968-2444

PiD - Psychotherapie im Dialog 2020; 21: 62-66

(c) Georg Thieme Verlag KG Stuttgart · New York

ISSN 1438-7026 